\title{
Acute pulmonary edema as clinical presentation of a peripartum cardiomyopathy in a very young patient
}

\author{
Riccardo Bentivegna, Egle Corrado, Giuseppe Coppola, \\ Emanuele Grassedonio, Salvatore Novo
}

\begin{abstract}
We present a case of a 20 -year-old woman at 34th week of pregnancy, hospitalized because of the risk of premature delivery. Because of a progressive worsening of clinical conditions with dyspnoea and desaturation, an emergency caesarean section with healthy fetal extraction was necessary. In the immediate post-operative period there was a sudden worsening of dyspnea with hypoxic-hypercapnic respiratory failure and severe pulmonary edema, with subsequent endotracheal intubation and mechanical ventilation. Echocardiography showed left atrial and ventricular dilatation, global hypokinesia with reduced systolicfunction (EF $30 \%)$, moderate mitral regurgitation with symmetrical tethering of the mitral leaflets and pericardial effusion. Cardiac magnetic resonance showed a noninflammatory pathway and was helpful to provide information in the differential diagnosis, so after a massive therapy we progressively observed
\end{abstract}

Riccardo Bentivegna1, Egle Corrado1, Giuseppe Coppola1, Emanuele Grassedonio ${ }^{2}$, Salvatore Novo ${ }^{1}$

Affiliations: ${ }^{1}$ Division of Cardiology, Regional Reference Centre for Diagnosis and Care of Heart Failure, Centre for the Early Diagnosis of Preclinical Atherosclerosis and for Secondary Prevention of Cardiovascular Diseases, Department of Internal Medicine and Cardiovascular Disease, University Hospital "Paolo Giaccone", Palermo, Italy; "Department of Radiological Sciences, University Hospital "Paolo Giaccone", Palermo, Italy.

Corresponding Author: Dr. Riccardo Bentivegna, Via Alcide De Gasperi n¹97, Sciacca (AG), 92019, Italy; Email: mich. bentivegna@alice.it

Received: 26 November 2017

Accepted: 11 January 2018

Published: 20 March 2018 a complete stabilization of the hemodynamic clinical picture, normalization of cardiac troponin and NT-proBNP levels and final full cardiac recovery. The early signs and symptoms of heart failure in peripartum cardiomyopathy may mimic physiological changes occurring during/after pregnancy, delayed diagnosis may occur. Although most patients with peripartum cardiomyopathy improve with medical therapy, nearly a third of all patients develop a worsening heart failure, and may also be associated with severe and lasting complications including cardiogenic shock and death.

Keywords: Acute pulmonary edema, Heart failure, Peripartum cardiomyopathy, Pregnancy

\section{How to cite this article}

Bentivegna R, Corrado E, Coppola G, Grassedonio E, Novo S. Acute pulmonary edema as clinical presentation of a peripartum cardiomyopathy in a very young patient. Int $\mathrm{J}$ Case Rep Images 2018;9:100896Zo1RB2018.

Article ID: 100896Zo1RB2018

$* * * * * * * * *$

doi: 10.5348/100896Zo1RB2018CR

\section{INTRODUCTION}

Peripartum cardiomyopathy (PPCM) is defined as a non-familial form of peripartum heart failure characterized as an idiopathic cardiomyopathy presenting with heart failure secondary to left-ventricular systolic 


\section{EDORIUM Journals}

dysfunction towards the end of pregnancy or in the months following delivery, where no other cause of heart failure is found. The left ventricle (LV) may not be dilated but the ejection fraction (EF) is nearly always reduced below 45\%. PPCM is considered an independent disease, whose diagnosis relies on its relation to pregnancy and the exclusion of other cardiomyopathies [1].

The early signs and symptoms of heart failure in PPCM patients may mimic physiological changes occurring during/after pregnancy, it might result in delayed diagnosis. The differential diagnosis of acute PPCM includes myocarditis, pre-existing cardiomyopathy, valve disease, or congenital heart disease [2].

The etiology of PPCM is still unknown, and many potential causes have been proposed but not proven. These include viral myocarditis, abnormal immune response to pregnancy, abnormal response to increased hemodynamic burden of pregnancy, hormonal abnormalities, excessive prolactin production, malnutrition, low selenium levels, stress-activated cytokines, inflammation and apoptosis, in addition to genetic factors [3-4].

Strong associations have been shown between PPCM and older maternal age, history of hypertension, multiple pregnancies, and African American background.

\section{CASE REPORT}

A 20-year-old Italian woman at the 34th week of pregnancy was hospitalized at the gynecology department of our hospital, because of the risk of premature delivery. Until hospitalization was a pregnancy with regular course (negative virological examination, hematochemical routine tests and morphological ultrasound). She had a history of partial epilepsy and two previous pregnancies (the first one without any complications and two years later a spontaneous abortion in the third month).

On admission she had a severe Sideropenic anemia (RBC 2.31 x10 $/ \mathrm{mL}$, Hgb $6.1 \mathrm{~g} / \mathrm{dL}$, sideraemia $16 \mathrm{mg} / \mathrm{dL}$, ferritin $6.8 \mathrm{ng} / \mathrm{dL}$ ) and low levels of C-reactive protein (4.7 mg/L), blood pressure was $105 / 70 \mathrm{mmHg}$, arterial oxygen saturation was $98 \%$ and 12-lead ECG showed sinus tachycardia with diffuse alterations of repolarization. In the following two days there was a progressive worsening of clinical conditions, with dyspnoea and desaturation, and on the third day an emergency caesarean section was performed in spinal anesthesia without intraoperative complications and with healthy fetal extraction.

In the immediate post-operative period, there was a sudden worsening of dyspnea with hypoxic-hypercapnic respiratory failure and clinical picture of severe pulmonary edema (with extensive pleural effusion and rales up to the lungs apexes). She was therefore transferred to the intensive care unit, was performed endotracheal intubation and connected to mechanical ventilation in assisted/controlled mode.

Echocardiography showed a slight dilated LV with normal wall thickness; global hypokinesia with reduced systolic function (EF 30\%); left atrial dilatation; moderate mitral regurgitation with symmetrical tethering of the mitral leaflets, slight anterior pericardial effusion (Figures 1A, 1B).

It was set diuretic therapy with endovenous infusion of furosemide, antibiotic therapy with levofloxacin and piperacillin/tazobactam, ionic correction of acidosis (sodium bicarbonate) and hydro-electrolytic balance, antithrombotic prophylaxis with enoxaparin and low dose of ramipril and aldactone. The investigation for common pathogens responsible for myocardial injury (legionella, chlamydia, mycoplasma, pneumococcus, coxsackievirus) was negative.

Following the progressive improvement in clinical conditions, our patient was extubated successfully and transferred to cardiology unit with an initial improvement of systolic cardiac function (EF 45\%) and a pathological ECG with Q-waves in V1-V2, high-voltage positive $\mathrm{T}$-waves in inferior leads and V3-V6 (Figure 2). Blood test demonstrated elevated levels of cardiac troponin I (225 $\mathrm{ng} / \mathrm{L})$ and NT-proBNP (759 pg/mL), normal creatinine $(0.4 \mathrm{mg} / \mathrm{dL})$, increase in hemoglobin levels $(10.8 \mathrm{~g} / \mathrm{dL})$ and sideraemia $(48 \mu \mathrm{g} / \mathrm{dL})$.

Cerebral and thoracic computed tomography (CT) excluded intra and extra-axial hemorrhages, recent ischemic lesions and pulmonary embolism, confirming instead a severe acute pulmonary edema (Figures 3(A and $\mathrm{B})$.

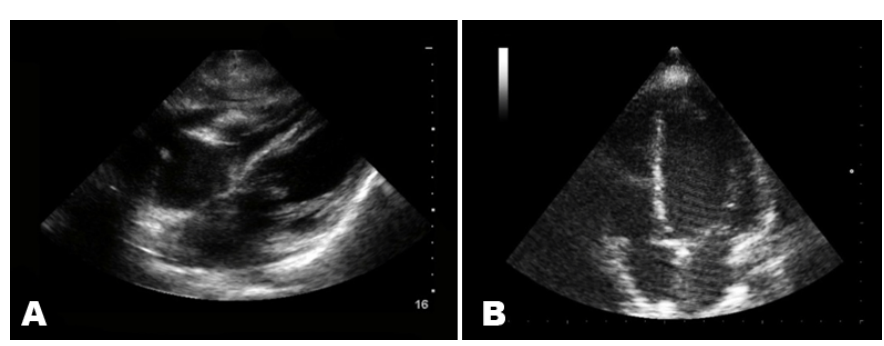

Figures 1: (A) 2-D transthoracic echocardiogram in subcostal view with pericardial effusion during acute phases, (B) apical four-chamber view.

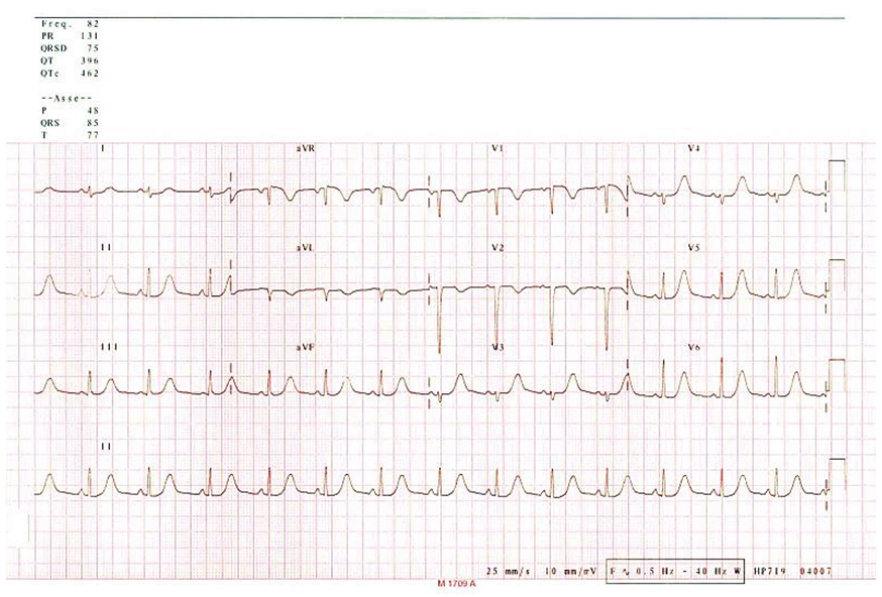

Figure 2: Pathological ECG with Q-waves and high-voltage positive T-waves. 


\section{EDORIUM Journals}

Cardiac magnetic resonance imaging (MRI) showed a global hypokinesia with mild reduction in left ventricular systolic function (EF 46\%), slight pericardial and pleural effusion, mitral regurgitation. In the T2-weighted sequences, no areas of hyperintensity of the signal, referable to myocardial edema. In the sequences acquired immediately after the administration of contrast media ev, no areas of hypointensity of the signal with subendocardial extension, referable to defects of perfusion at rest. In late sequences no areas of intra-myocardial hyperintensity, referable to fibrosis (Figure $4 \mathrm{~A}-\mathrm{C}$ ).

Standard heart failure therapy (ramipril, bisoprolol, furosemide and spironolactone) and antibiotic therapy was continued with a complete stabilization of the hemodynamic clinical picture, normalization of cardiac troponin and NT-proBNP levels, improvement of left ventricular function to serialized echocardiographic controls (EF 52\%) and discharge at the 13th day of hospitalization.

The young patient was visited a month after discharge into perfect clinical conditions and a full cardiac recovery by echocardiography: left sides with normal sizes; normal systolic function (EF 65\%); slight mitral regurgitation (reduced), absence of pericardial effusion.

For this reason we opted for a gradual discontinuation of therapy with a frequent monitoring of LV function every 3-6 months for the follow years.

\section{DISCUSSION}

The clinical presentation of patients with PPCM is similar to those with other forms of systolic HF secondary to cardiomyopathy, but may be highly variable. Early signs and symptoms of PPCM may often mimic normal physiological findings of pregnancy and include pedal oedema, weight gain, physical discomfort, dyspnoea on exertion, orthopnoea, paroxysmal nocturnal dyspnoea, and persistent cough. Additional signs and symptoms experienced in PPCM include abdominal discomfort secondary to hepatic congestion, dizziness, praecordial pain, palpitations, and in the later stages postural hypotension, third heart sound and pulmonary rales [1].

For this reason, and because of the low incidence of this condition, the diagnosis of PPCM is often missed or delayed, allowing the development of preventable complications.

Echocardiography in PPCM shows variable degrees of LV dilatation with moderate to severe depression of systolic function, right ventricular and bi-atrial dilatation and moderate to severe mitral and tricuspid regurgitation with increased pulmonary pressures.

Cardiac magnetic resonance imaging (MRI) has an important role in prognostic stratification, by the assessment of LV size, function and contractile reserve, the detection of mural thrombi or myocardial fibrosis and finallythepresenceorabsenceofmyocardiallategadolinium enhancement [5]. By distinguishing inflammatory (late

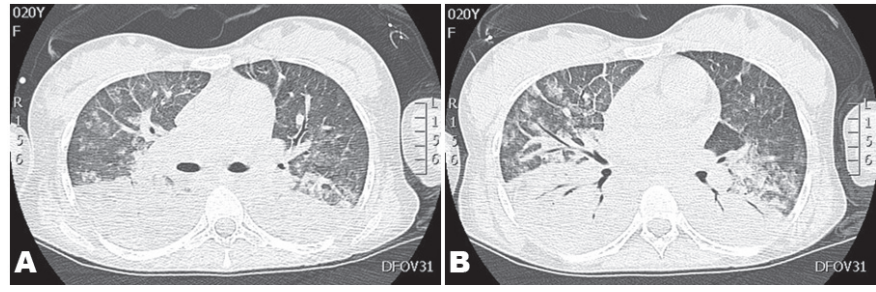

Figures 3 (A and B): Chest CT with pleural effusion and parenchymal consolidation, interstitial edema, alveolar edema (ground glass), enlargement of pulmonary segmental arteries.
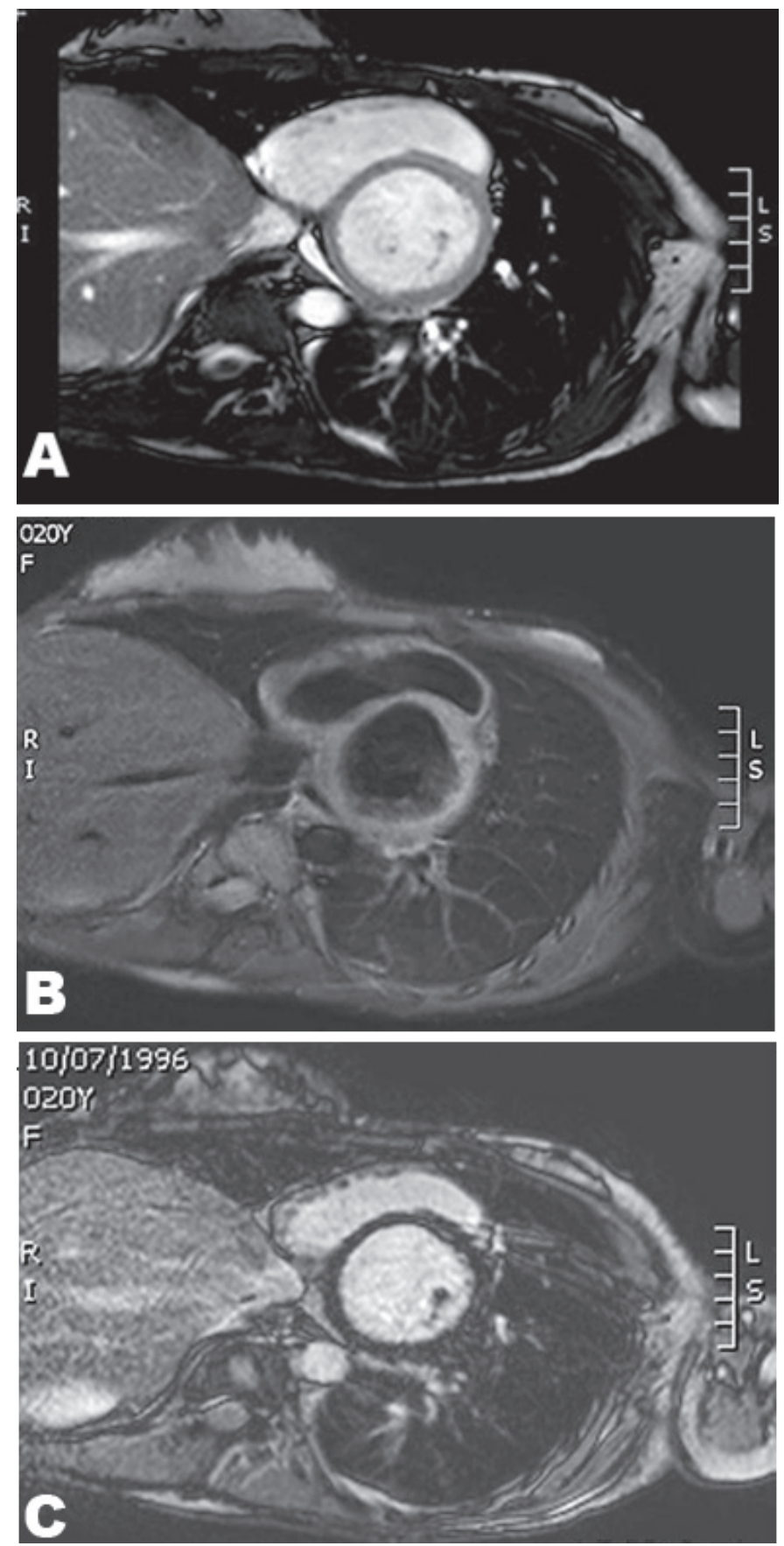

Figures 4: (A) cardiac MRI obtained at end diastole showing basal short-axis slices, (B) normal T2-weighted signal, (C) absence of delayed gadolinium enhancement. 


\section{EDORIUM Journals}

gadolinium enhancement in T1-weighted sequences ; hypersignal in T2-weighted sequences) and noninflammatory (absence of enhancement and hypersignal) forms of PPCM, cardiac MRI can be helpful at initial presentation to consider appropriate physiopathologic hypothesis and to guide further etiologic investigations. It can also guide therapeutic options, being in favor of using immunosupressor agents in inflammatory forms of PPCM and provide critical information in the differential diagnosis of myocarditis, Takotsubo or ischemic cardiomyopathy [6-7].

Although the prognosis is more favorable than in other types of cardiomyopathies, PPCM may be associated with severe and lasting complications including severe HF, pulmonary edema, cardiogenic shock, cardiopulmonary arrest secondary to HF or arrhythmias, thromboembolic complications, and death. Predictors of complications are left ventricular ejection fraction (LVEF) $<25 \%$, nonCaucasian ethnic background, and delay of diagnosis. [8]

A number of factors have been shown to be associated with a higher likelihood of recovery, including LV diastolic dimension ( $<55$ to $60 \mathrm{~mm}$ ) and systolic function (LVEF $<30 \%$ to $35 \%$ and fractional shortening $<20 \%$ ) at the time of diagnosis, lack of troponin elevation, a lower level of plasma BNP, absence of LV thrombus, breast-feeding, diagnosis after the delivery, and non-African American ethnicity [2].

Currently, PPCM is treated according to the ESC guidelines for heart failure in pregnancy (e.g. hydralazine and nitrates). After delivery, standard therapy for heart failure is recommended in PPCM including beta-blockers, ACE-inhibitors/AT1-blockers, intravenous inotropes, intravenous and oral vasodilators, mineralocorticoid receptor antagonists (MRA), and diuretics [9-10].

Although most patients with PPCM improve with medical therapy, nearly a third of all patients develop worsening heart failure. A general agreement among experts suggests continued therapy with standard heart failure medications for a minimum of 12 months [11].

\section{CONCLUSION}

Pregnancy, delivery, and the peripartum period provide a challenge to the entire female organism, therefore understanding normal pregnancy is important for the timely recognition of cardiovascular pathologies, to distinguish the peripartum discomfort in healthy women from signs of cardiovascular disease. This is a major challenge for physicians and patients, and may explain why diagnosis of PPCM is frequently delayed and still underestimated.

Gradual discontinuation with frequent monitoring of $\mathrm{LV}$ function is reasonable in patients with complete recovery of LV systolic function (LVEF > 55\%) and normal LV size, contrarily standard heart failure therapy should be continued in patients with persistently reduced LVEF for several years or even lifelong.

\section{REFERENCES}

1. Sliwa K, Hilfiker-Kleiner D, Petrie MC, et al. Current state of knowledge on aetiology, diagnosis, management, and therapy of peripartum cardiomyopathy: A position statement from the heart failureassociation of the European society of cardiology working group on peripartum cardiomyopathy. Eur J Heart Fail 2010 Aug;12(8):767-78.

2. Elkayam U. Clinical characteristics of peripartum cardiomyopathy in the United States: Diagnosis, prognosis, and management. J Am Coll Cardiol 2011 Aug 9;58(7):659-70.

3. Ntusi NB, Mayosi BM. Aetiology and risk factors of peripartum cardiomyopathy: A systematic review. Int J Cardiol 2009 Jan 9;131(2):168-79.

4. Sliwa K, Förster O, Libhaber E, et al. Peripartum cardiomyopathy: inflammatory markers as predictors of outcome in 100 prospectively studied patients. Eur Heart J 2006 Feb;27(4):441-6.

5. Assomull RG, Prasad SK, Lyne J, et al. Cardiovascular magnetic resonance, fibrosis, and prognosis in dilated cardiomyopathy. J Am Coll Cardiol 2006 Nov 21;48(10):1977-85.

6. Mouquet F, Lions $\mathrm{C}$, de Groote $\mathrm{P}$, et al. Characterisation of peripartum cardiomyopathy by cardiac magnetic resonance imaging. Eur Radiol 2008 Dec;18(12):2765-9.

7. Mahrholdt H, Wagner A, Judd RM, Sechtem U, Kim RJ. Delayed enhancement cardiovascular magnetic resonance assessment of non-ischaemic cardiomyopathies. Eur HeartJ 2005Aug;26(15):146174.

8. Goland S, Modi K, Bitar F, et al. Clinical profile and predictors of complications in peripartum cardiomyopathy. J Card Fail 2009 Oct;15(8):645-50.

9. Regitz-Zagrosek V, Blomstrom-Lundqvist C, Borghi $\mathrm{C}$, et al. ESC Guidelines on the management of cardiovascular diseases during pregnancy: The task force on the management of cardiovascular diseases during pregnancy of the European society of cardiology (ESC). Eur Heart J 2011 Dec;32(24):314797.

10. Lindenfeld J, Albert NM, Boehmer JP, et al. Executive summary: HFSA 2010 comprehensive heart failure practice guideline. J Card Fail 2010;16(6):475-539.

11. Hilfiker-Kleiner D, Haghikia A, Nonhoff J, Bauersachs J. Peripartum cardiomyopathy: Current management and future perspectives. Eur Heart J 2015 May 7;36(18):1090-7.

\section{$* * * * * * * * *$}

\section{Author Contributions}

Riccardo Bentivegna - Substantial contributions to conception and design, Acquisition of data, Analysis and interpretation of data, Drafting the article, Revising it critically for important intellectual content, Final approval of the version to be published

Egle Corrado - Substantial contributions to conception and design, Acquisition of data, Analysis and interpretation of data, Drafting the article, Revising 
it critically for important intellectual content, Final approval of the version to be published

Giuseppe Coppola - Substantial contributions to conception and design, Acquisition of data, Analysis and interpretation of data, Drafting the article, Revising it critically for important intellectual content, Final approval of the version to be published

Emanuele Grassedonio - Substantial contributions to conception and design, Acquisition of data, Analysis and interpretation of data, Drafting the article, Revising it critically for important intellectual content, Final approval of the version to be published

Salvatore Novo - Substantial contributions to conception and design, Acquisition of data, Analysis and interpretation of data, Drafting the article, Revising it critically for important intellectual content, Final approval of the version to be published

\section{Guarantor of Submission}

The corresponding author is the guarantor of submission.

\section{Source of Support}

None

\section{Consent Statement}

Written informed consent was obtained from the patient for publication of this report.

\section{Conflict of Interest}

Authors declare no conflict of interest.

\section{Copyright}

(C) 2018 Riccardo Bentivegna et al. This article is distributed under the terms of Creative Commons Attribution License which permits unrestricted use, distribution and reproduction in any medium provided the original author(s) and original publisher are properly credited. Please see the copyright policy on the journal website for more information.
Access full text article on other devices

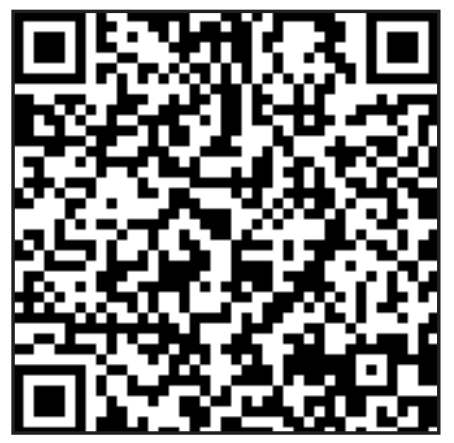

Access PDF of article on other devices

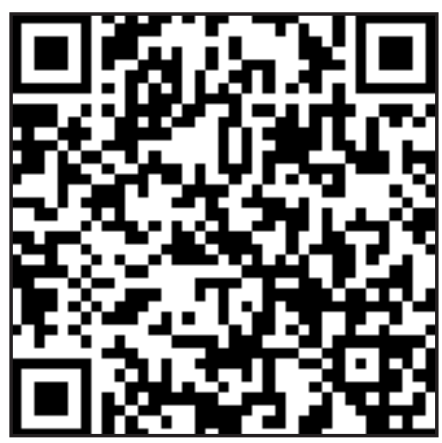

